\title{
Analysis of Charge Storage in Polysilicon Contacts
}

\author{
D. Bardés and R. Alcubilla \\ Departament d'Enginyeria Electrònica, ETSETB - UPC \\ P.O.Box 30002, E-08034 Barcelona, SPAIN
}

\begin{abstract}
We present the use of a commercial 2D device simulator, MEDICI [1], to analyze a polysilicon emitter contact. By using a 2-box model we adjust simultaneously the effective recombination velocity and the stored charge in the polysilicon layer.
\end{abstract}

\section{Introduction}

Polysilicon emitter transistors have been a main topic in device research during the last years [2], [3]. The progressive scaling-down of both horizontal and vertical dimensions enhances the interest of an accurate simulation of the static and dynamic characteristics of this kind of contacts.

Simulation of these contacts is usually done [4] by using an effective recombination velocity as a boundary condition. The stored charge can be then considered through a correction in the emitter transit time $\tau_{e}$ [5].

$$
\tau_{e}=\tau_{e s} \cdot\left(1+\frac{Q_{e p}}{Q_{e s}}\right)
$$

In most $2 \mathrm{D}$ simulators as MEDICI, polysilicon contacts are simulated through the use of virtual semiconductor parameters in the polysilicon layer. This allows to fit the DC characteristics of the whole bipolar transistor [4].

In this work we propose a two layer model that can be easily implemented through MEDICI allowing to adjust the DC characteristics and at the same time to evaluate the stored charge in the polysilicon layer.

\section{Simulation method}

Classically, the simulation of polysilicon emitter contacts trough MEDICI is done providing an equivalent boundary condition [6] to the device, that considers an effective surface recombination velocity:

$$
J=q \cdot S_{\text {eff }} \cdot p
$$


$S_{\text {ef }}$ is obtained by modifying the minority carrier mobility and lifetime in the region corresponding to the polysilicon. Solving the diffusion equation in this zone with these modified parameters leads to the desired effective recombination velocity.

$$
S_{e f f}=\frac{D_{p}}{L_{p}} \operatorname{coth} \frac{W_{P o l y}}{L_{p}}
$$

Where $D_{p}$ is the modified diffusion coefficient for holes, $L_{p}$ the modified diffusion length and $W_{\text {Poly }}$ is the polysilicon layer width. Although this procedure can be useful for DC conditions, we have no mean to control the stored charge in the poly. As this charge may significantly modify the forward transit time in scaled down structures [5], it is worthy to have a realistic estimation for the stored charge in the poly, and, as a consequence, for $\tau_{\text {poly }}$.

Our proposal, based on the box model [6], is to consider two regions to simulate the contact. One corresponding to the thin oxide layer (or a grain boundary, if there is no oxide present), and another to the rest of the contact. The properties of this oxide layer mostly determine the stored charge in the poly.

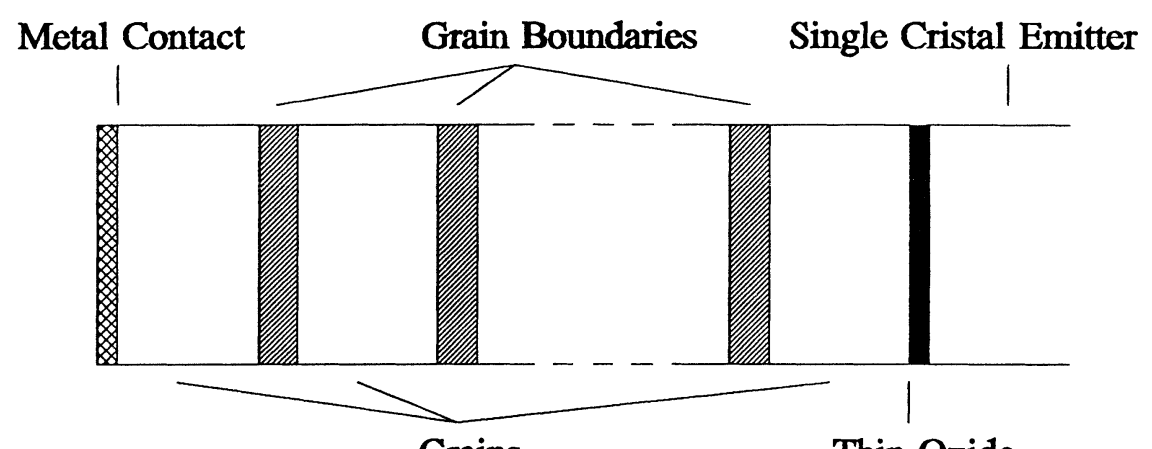

Figure 1: Scheme of a polysilicon emitter, box model.

The parameters describing this two regions, i.e. the minority carrier lifetimes and mobilities, are found from the equations and parameters given in [6] for the hole transport.

For the oxide layer, the effective diffusion coefficient, $D_{e f f}$, and the effective diffusion length, $L_{e f f}$ are calculated through.

$$
\begin{gathered}
L_{e f f}=\frac{d_{o x}}{\arg \cosh \frac{S_{o x}+T_{o x}}{T_{o x}}} \\
D_{e f f}=T_{o x} \cdot L_{e f f} \cdot \sinh \frac{d_{o x}}{L_{e f f}}
\end{gathered}
$$

$T_{o x}$ is a coefficient related to the tunneling trough the oxide, and depends [6] on the hole potential barrier, $\chi_{h}$ and the hole effective mass in the oxide, $m_{h}^{*}, S_{o x}$ a surface recombination velocity located at the two edges of the thin oxide layer, and $d_{o x}$ is the oxide layer width. 
The second region, which models the polysilicon, can be described classically through the modified mobility method mentioned above.

In this way good estimations for the charge in the poly can be obtained. If more accuracy is desired, it is easy to extend the method to consider the polysilicon grains and grain boundaries in different regions instead of joining them in a single region. The grain boundary zones are described in [6] through expressions (4) and (5) using a $T_{g b}$ (a transport coefficient in the grain boundary), and $S_{g b}$, for recombination, instead of $T_{o x}$ and $S_{o x}$.

\section{Results}

We have used the parameters shown in table $I$ in equations (3) to (5) to simulate a polysilicon emitter with MEDICI, and to perform the analytical calculations. In Figures 2 and 3 we compare the obtained results with those found through analytical expressions.

\begin{tabular}{lcc}
\hline Polysilicon layer thickness & WPoly & $0.4 \mu \mathrm{m}$ \\
Hole transport parameters in grain boundaries & $\mathbf{T}_{\mathrm{gb}}$ & $2.510^{5} \mathrm{~cm} / \mathrm{s}$ \\
& $\mathbf{S}_{\mathrm{gb}}$ & $7.510^{4} \mathrm{~cm} / \mathrm{s}$ \\
Grain boundaries thickness & $\mathbf{d g b}_{\mathrm{gb}}$ & $20 \AA$ \\
Recombination velocity at the oxide edges & $\mathrm{S}_{\mathrm{Ox}}$ & $1.510^{3} \mathrm{~cm} / \mathrm{s}$ \\
Hole effective mass in the oxide & $\mathrm{m}_{\mathrm{h}}^{*}$ & $0.42 \mathrm{~m}$ \\
Hole potential barrier in the oxide & $\chi_{\mathrm{h}}$ & $1 \mathrm{eV}$ \\
Hole diffusion coefficient in the grains & $\mathrm{D}_{\mathrm{p}}$ & $3.28 \mathrm{~cm}^{2} / \mathrm{s}$ \\
Hole diffusion length in the grains & $\mathrm{L}_{\mathrm{p}}$ & $0.812 \mu \mathrm{m}$ \\
Donor concentration in the polysilicon & $\mathrm{N}_{\text {dpoly }}$ & $710^{19} \mathrm{~cm}^{-3}$ \\
\hline
\end{tabular}

Table 1: Values used for the calculations

We can see in Figure 2 the stored charge in the poly as a function of the oxide layer thickness. Results obtained with the classical method and through the analytical expressions are shown for comparison.

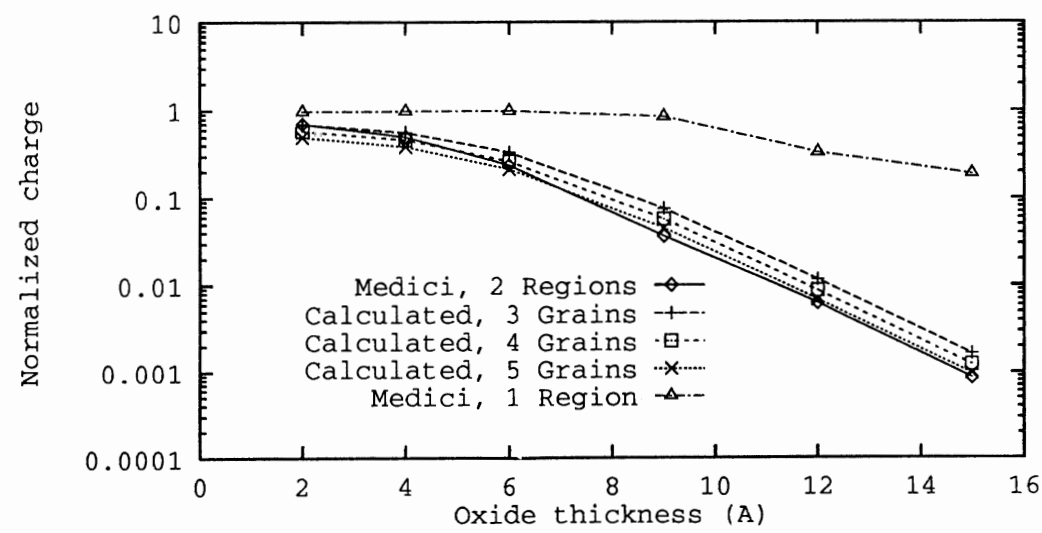

Figure 2: Integrated charge density as a function of oxide thickness. 


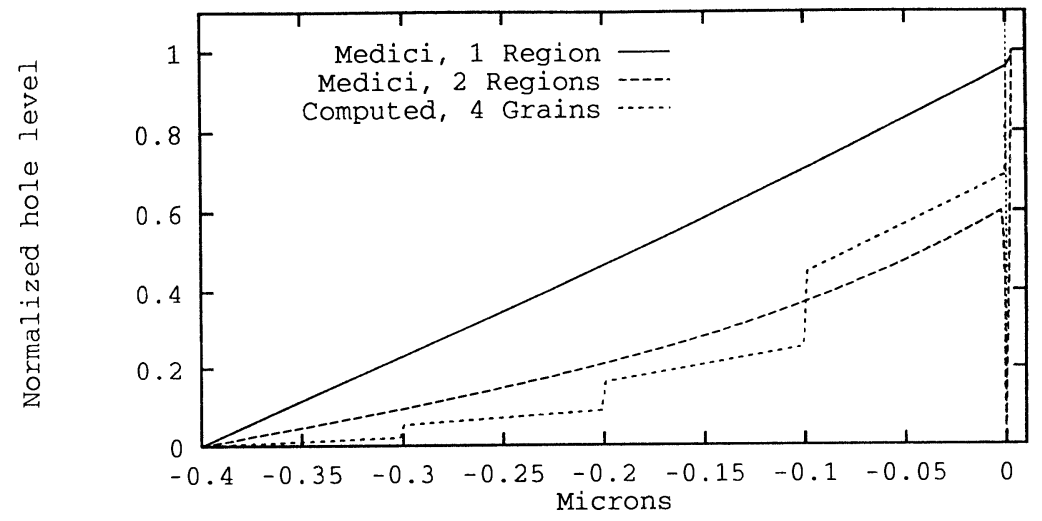

Figure 3: Hole concentration profile versus depth in polysilicon and oxide layers.

In Figure 3 the hole profile in the polysilicon layer is shown. Three cases are compared for an oxide thickness of $4 \AA$ : the obtained analitically in the case of 4 grains [5], and those found with MEDICI, through the proposed method and by using the classical one.

\section{Conclusions}

This work uses a numerical simulator, MEDICI, to evaluate the charge storage in the polysilicon layer of a polysilicon emitter bipolar transistor. The used method provides an estimation for the stored charge that agrees with previous results. If more accuracy is needed, a straightforward extension of the method has been proposed.

\section{References}

[1] Technology Modelling Associates, Inc., MEDICI User's Manual. March 1992

[2] G. L. Patton et al, 75-GHz $f_{t}$ SiGe-Base Heterojunction Bipolar Transistors, IEEE Electron Device Letters, p 171. 1990

[3] M. Nanba et al, A 64 GHz Si Bipolar Transistor Using In-Situ Phosphorus Doped Polysilicon Emitter Technology, IEDM Technical Digest, p 443. 1991

[4] P. Vande Voorde et al, Hybrid Simulation and Sensitivity Analysis for Advanced Bipolar Device Design and Process Developement, B.C.T.M. Technical Digest, $\mathrm{p}$ 114. 1990

[5] L. M. Castañer and S. Sureda, A Compact Charge Ratio Expression for the Emitter Delay of Polysilicon Emitter Bipolar Transistors, IEEE Transactions on Electron Devices, Submitted.

[6] Z. Yu et al, A Comprehensive Analytical and Numerical Model of Polysilicon Emitter Contacts in Bipolar Transistors, IEEE Transactions on Electron Devices, p 773.1984 